\title{
Caracterización del dolor en el paciente en hemodiálisis
}

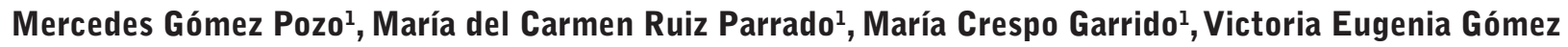 \\ López ${ }^{2}$, Rodolfo Crespo Montero ${ }^{1,2,3}$
}

${ }^{1}$ Departamento de Enfermería. Facultad de Medicina y Enfermería. Universidad de Córdoba. Córdoba. España

${ }^{2}$ Unidad de Gestión Clínica de Nefrología. Hospital Universitario Reina Sofía de Córdoba. Córdoba. España

${ }^{3}$ Instituto Maimónides de Investigación Biomédica de Córdoba. Córdoba. España

\section{Resumen}

Objetivos: El objetivo del presente estudio fue analizar la prevalencia y las características del dolor de los pacientes en hemodiálisis.

Material y Método: Se llevó a cabo un estudio observacional descriptivo y transversal en 134 pacientes (40\% mujeres y edad media de $68.05 \pm 13.77$ años) del área de influencia del Servicio de Nefrología del Hospital Reina Sofía de Córdoba. Para valorar las características del dolor se utilizó el Cuestionario de Dolor Español y Cuestionario Breve del Dolor. Además, se evaluó la dependencia y comorbilidad.

Resultados: El 69\% de los pacientes manifestaron padecer dolor, frente a un $31 \%$ que no tenían. El dolor percibido como más doloroso fue el de origen músculo-esquelético y los calambres, con una prevalencia del $36 \%$ y $24 \%$, respectivamente. El $64 \%$ del dolor se localizó en miembros inferiores, seguido del localizado en la espalda. Respecto a la Intensidad Máxima, del peor dolor padecido en la última semana, el $66 \%$ lo calificó como severo, mientras que en el momento de la entrevista un 38\% no tenía dolor. El estado de ánimo y el descanso fueron las actividades menos afectadas por el dolor, con una mediana de 5 .

Conclusiones: La prevalencia del dolor es alta en los pacientes estudiados, siendo el de origen músculo-esquelético el más frecuente y localizado principalmente en miembros inferiores; además, el dolor tiene una repercusión directa en la mayoría de las actividades de

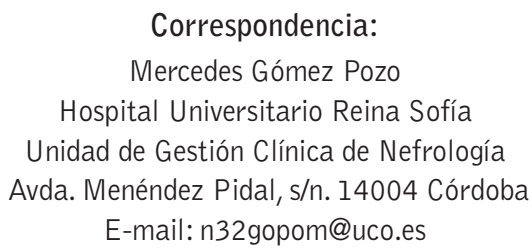

la vida diaria de los pacientes. La principal causa de dolor relacionada con el procedimiento de la hemodiálisis son los calambres musculares.

PALABRAS CLAVE: dolor; dolor crónico; hemodiálisis; enfermedad renal crónica avanzada.

-

Characterization of pain in the hemodialysis patient

\begin{abstract}
Objective: The aim of the present study was to analyze the prevalence and characteristics of pain in hemodialysis patients.

Material and Method: A descriptive and cross-sectional observational study was carried out in 134 patients (40\% women and mean age of $68.05 \pm 13.77$ years) from the area of influence of the Nephrology Unit of the Reina Sofía University Hospital in Córdoba. To assess the characteristics of the pain, the Spanish Pain Questionnaire and the Brief Pain Questionnaire were used. In addition, dependency and comorbidity were evaluated.
\end{abstract}

Results: Sixty-nine percent of patients reported suffering pain, compared to $31 \%$ that they did not have. The pain perceived as most painful was that of musculoskeletal origin and cramps, with a prevalence of $36 \%$ and $24 \%$, respectively. $64 \%$ of the pain was located in the lower limbs, followed by the one located in the back. Regarding the maximum intensity, of the worst pain suffered in the last week, $66 \%$ rated it as severe, while at the time of the interview $38 \%$ did not have pain. The mood and rest were the activities least affected by the pain, with a median of 5 . 
Conclusions: The prevalence of pain is high in patients studied, being the musculoskeletal origin the most frequent and located mainly in the lower limbs; In addition, pain has a direct impact on most activities of patients' daily lives. Muscle cramps are the main cause of pain related to the hemodialysis procedure.

KEYWORDS: pain; chronic pain; characerization of pain; hemodialysis; advanced chronic kidney disease.

\section{Introducción}

El dolor constituye un problema de salud pública de primera magnitud, ya que interfiere directamente en el bienestar y la calidad de vida de las personas, con gran repercusión social y económical-3. La Asociación Mundial para el Estudio del Dolor (IASP) define el dolor como "una experiencia sensorial y emocional desagradable, asociada con un daño tisular, real o potencial, o descrita en términos de dicho daño ${ }^{\prime \prime 4}$.

Su expresión atiende a un patrón particular e individualizado según la persona que lo padece, así como a las diferentes variables psicosociales y culturales ${ }^{5}$. Sin embargo, la evidente variabilidad de su percepción no exime de su estudio, dado que es un problema que afecta a un gran número de personas y puede ser limitante en muchas de ellas 6 . Según un estudio reciente, el $17,25 \%$ de la población española sufre algún tipo de dolor ${ }^{7}$.

Una población especialmente sensible a padecer dolor son los pacientes con Enfermedad Renal Crónica Avanzada $(E R C A)^{8-12}$. Según el Registro de la Sociedad Española de Nefrología de 2016, la prevalencia global de la ERCA en España es de 1233,5 pacientes por millón de población, de los cuales el $42.2 \%$ está en programa de Hemodiálisis (HD). A esto hay que añadir, que más del $60 \%$ son mayores de 65 años ${ }^{13}$.

El paciente en HD, además de mayor, soporta otras patologías concomitantes presentando una elevada comorbilidad asociada, entre la que destaca la diabetes, la enfermedad coronaria, la enfermedad vascular periférica, etc ${ }^{14}$. Todo esto, unido al tiempo que permanecen en tratamiento renal sustitutivo, condiciona que el paciente en HD sea un sujeto altamente susceptible de padecer dolor ${ }^{15,16}$.

En este sentido, según diferentes estudios la prevalencia del dolor en los pacientes en HD se sitúa entre el $50-70 \%{ }^{17-19}$, indicando además, que el $82 \%$ de estos pacientes califica este dolor con una intensidad de mo- derada a severa y un manejo inefectivo ${ }^{20}$; siendo el dolor músculo-esquelético el más frecuente, seguido del dolor relacionado con la técnica dialítica, el de origen isquémico y neuropático ${ }^{19,21}$.

Por consiguiente, el paciente en HD está abonado a padecer algún tipo de dolor, bien sea inherente a las alteraciones osteoarticulares propias de la ERCA, relacionado con la técnica sustitutiva o bien debido a la comorbilidad añadida. De ahí, que consideremos imprescindible el análisis del tipo, grado y localización del dolor que padecen estos pacientes, para su mejor conocimiento y manejo.

Por consiguiente, el objetivo general de este estudio fue evaluar la prevalencia y las características del dolor de los pacientes en HD, con los siguientes objetivos específicos:

- Describir la localización y el origen más frecuente del dolor del paciente en HD.

- Caracterizar el dolor relacionado con el procedimiento de HD.

- Valorar la repercusión del dolor en las actividades de la vida diaria (AVD) del paciente en HD.

\section{Material y Método}

\section{Ámbito del estudio}

El estudio se llevó a cabo en el área de influencia del Servicio de Nefrología del Hospital Universitario Reina Sofía de Córdoba, en 3 centros de diálisis: 2 públicos y 1 concertado; y se ha llevado a cabo entre los meses de marzo y mayo de 2017.

\section{Diseño}

Se realizó un estudio observacional, descriptivo y transversal.

\section{Población y muestra}

La población de estudio fueron pacientes con ERCA en programa de HD periódicas. Se realizó un muestreo no probabilístico intencional. Se les pidió autorización al total de pacientes de los 3 centros y, tras descartar a los que no aceptaron participar, se obtuvo una muestra total de 134 pacientes.

\section{Criterios de inclusión}

Los criterios de inclusión para la participación en el estudio fueron: pacientes de ambos sexos que estuvieran 
en tratamiento de $\mathrm{HD}$, con capacidad para comunicarse con la entrevistadora y que aceptaran participar en la investigación.

\section{Variables del estudio}

Variables de resultado: tipo, características y grado de dolor.

Otras variables fueron: edad, género, grado de dependencia e índice de comorbilidad.

\section{Instrumentos de medida}

Previamente a la aplicación de los diferentes cuestionarios, la entrevistadora preguntaba a los pacientes si habían experimentado dolor en la última semana. En caso afirmativo, se procedió a la cumplimentación de los cuestionarios pidiéndole al entrevistado que supiera localizar y describir de la manera más completa su dolor.

Para evaluar el dolor se utilizaron dos cuestionarios validados: el Cuestionario Breve del Dolor (CBD); versión española y validada del Brief Pain Inventory, y el Cuestionario de Dolor Español (CDE), obtenido de la versión inglesa McGuill Pain Questionnaire.

EI CBD recoge información sobre las distintas intensidades del dolor, la medicación y su manejo; y, hasta qué punto el dolor afecta a los pacientes en sus actividades diarias. Por su parte, el CDE evalúa: el valor de Intensidad Total del dolor, el Valor de Intensidad Actual, además de una Escala Visual Analógica (EVA) de 0 10. La Intensidad Total, con valores entre 0 y 14, se obtiene de la suma de las siguientes dimensiones: Intensidad Sensorial (0-7), Intensidad Afectiva (0-6) e Intensidad Evaluativa (0-1). El Valor de Intensidad Actual se obtiene de una escala tipo Likert de 0 a 5.

Para valorar la capacidad para realizar las actividades básicas de la vida diaria (ABVD) de manera independiente se utilizó el Índice de Katz.

Para analizar la comorbilidad se utilizó el Índice de Comorbilidad de Charlson (ICC).

\section{Recogida de datos}

Los datos se obtuvieron en los respectivos centros cuando los pacientes asistían a la sesión de HD, durante las dos primeras horas de la misma. Se escogió este tramo horario para evitar interferencias con la percepción dolorosa y el malestar que suelen presentar estos pacientes en la última hora de la sesión.
La cumplimentación de los cuestionarios la realizó una única entrevistadora. Se explicó el objetivo del estudio a cada uno de los pacientes y se les pidió el consentimiento informado. Se garantizó en todo momento la confidencialidad de los datos recogidos y el anonimato de éste. El estudio se inició después de obtener la aprobación del Comité de Ética e Investigación Provincial.

\section{Análisis estadístico}

Se realizó una distribución de frecuencias para las variables cualitativas y la media \pm la desviación estándar de la media para variables cuantitativas. También se realizaron asociaciones entre variables utilizando test paramétricos o no, según seguían una distribución normal 0 no. Se aceptó significación estadística para $p<0.05$. Los datos se analizaron con el paquete estadístico SPSS 15.0 para Windows.

\section{Resultados}

La muestra del estudio estuvo formada por 134 pacientes, $81(60 \%)$ hombres y $53(40 \%)$ mujeres, con una edad media de $68.05 \pm 13.77$ años, y una mediana de tiempo en HD de 48 meses con un rango intercuartil (RI) de 73 meses. El total de pacientes se distribuyó por centros de la siguiente forma: Unidad Hospitalaria (UH) 28 (21\%), Centro Periférico Público (CPP) 43 (32\%) y Centro Concertado (CC) 63 (47\%).

Cuando se analizó la capacidad de los pacientes para realizar las ABVD de forma independiente o no, según el índice de Katz, el 10\% presentaba una incapacidad severa, el $23 \%$ una incapacidad moderada y el $67 \%$ una incapacidad leve 0 ausencia de incapacidad.

En cuanto al ICC de toda la muestra, se obtuvo una mediana de 6 con un RI de 2. Cuando se relacionaron las distintas variables, se encontró una correlación estadísticamente significativa entre la edad, el ICC $(r=0.501$, $p<0.01)$ y el Índice de Katz $(r=0.392, p<0.01)$; y entre el ICC y el Índice de Katz $(r=0.260, p<0.01)$.

En cuanto a la prevalencia del dolor, 93 pacientes (69\%) dijeron PADECER dolor (de los cuales el $39 \%$ eran diabéticos) frente a 41 pacientes (31\%) que manifestaron NO haber tenido dolor (de los cuales el 29\% eran diabéticos), en la última semana. La edad media de los pacientes que presentaron dolor fue mayor que la de los que no tenían dolor $(68.76 \pm 14.33$ frente a $66.44 \pm 12.42$ ), aunque las diferencias no fueron significativas. 
De los tres centros estudiados, los pacientes que más prevalencia de dolor presentaron fueron la UH (89\%), seguida del CPP (67\%) y por último el CC $(62 \%)$.

En cuanto al tiempo en HD, el grupo que presentó dolor llevaba una mediana de 54 meses (RI 80) y el grupo que no presentó dolor 38 (RI 50), aunque las diferencias no fueron estadísticamente significativas. No se encontraron diferencias entre los pacientes con dolor o sin dolor en el ICC (mediana de 6 frente a 6 , ) ni en el Índice de Katz (1 frente 1, mediana). Sin embargo, cuando se analizó el ICC por separado entre ambos grupos, encontramos que en el grupo con dolor, el 55\% de los pacientes se encuentran por debajo de la mediana (6) y el $45 \%$ por encima de esta. Sin embargo, en el grupo sin dolor el $71 \%$ está por debajo de la mediana y el $29 \%$ está por encima.

Con respecto al Índice de Katz en los pacientes que presentaban dolor, el 56\% tenían Ausencia o Incapacidad Leve y el $44 \%$ tenían Incapacidad Moderada $(30 \%)$ o Severa (14\%). En cambio los que manifestaron no tener dolor, tan solo el $7 \%$ presentó una Incapacidad Moderada y el 93\% restante Ausencia o Incapacidad Leve.

Una vez analizada la muestra global, el análisis estadístico se centró en los pacientes que presentaron dolor $(n=93)$. Entre estos el $56 \%$ eran hombres y el $44 \%$ mujeres. Al comparar la Intensidad Total (del cuestionario CDE) de dolor en este grupo respecto al sexo, las mujeres presentaron mayor intensidad dolorosa que los hombres $(9.66 \pm 2.17$ frente a $8.33 \pm 2.0, p<0.003)$, igual que con la Intensidad Actual $(p<0.030)$ y la Intensidad Máxima del CBD $(p<0.044)$.

En la Tabla 1 se representan las causas y la localización del dolor; y como puede apreciarse, el dolor de origen músculo-esquelético fue el más prevalente seguido del relacionado con el procedimiento, en el que destacaron los calambres.

En relación a la localización del dolor, las extremidades fueron la parte más afectada; sobre todo los miembros inferiores. La espalda y el brazo de la fístula con porcentajes similares, fueron también focos de dolor importantes.

Las Tablas 2, 3 y 4 recogen los resultados del CBD.
Tabla 1. Causas y localización del dolor.

\begin{tabular}{|c|c|c|}
\hline Causas & & \\
\hline Músculo-esquelético & & $33(36 \%)$ \\
\hline \multirow[t]{2}{*}{ Relacionado con el acceso vascular } & & $11(12 \%)$ \\
\hline & Calambres & $22(24 \%)$ \\
\hline \multirow[t]{2}{*}{ Relacionado con el procedimiento } & Cefalea & $4(4 \%)$ \\
\hline & Dolor gastrointestinal & $2(2 \%)$ \\
\hline Isquémico & & $16(17 \%)$ \\
\hline Neuropático & & $2(2 \%)$ \\
\hline Otro & & $3(3 \%)$ \\
\hline \multicolumn{3}{|l|}{ Localización } \\
\hline Extremidades superiores e inferiores & & $9(10 \%)$ \\
\hline Miembros superiores & & $7(7 \%)$ \\
\hline Miembros inferiores & & $44(47 \%)$ \\
\hline Cabeza & & $4(4 \%)$ \\
\hline Espalda & & $15(16 \%)$ \\
\hline Brazo de la fístula arteriovenosa & & $14(15 \%)$ \\
\hline
\end{tabular}

Tabla 2. Intensidad, repercusión y percepción del dolor en las AVD.

\begin{tabular}{|c|c|c|c|c|c|}
\hline Intensidad & Leve & Moderada & Severa & Mediana & RI \\
\hline Máxima & $2(2 \%)$ & $30(32 \%)$ & $61(66 \%)$ & 8 & 2 \\
\hline Mínima & $70(75 \%)$ & $23(25 \%)$ & - & 3 & 7 \\
\hline Media & $24(27 \%)$ & $60(68 \%)$ & $4(5 \%)$ & 5 & 6 \\
\hline Actual & $68(73 \%)$ & $20(22 \%)$ & $5(5 \%)$ & 2 & 10 \\
\hline \multicolumn{6}{|c|}{ Repercusión en las AVD } \\
\hline \multicolumn{2}{|l|}{ Actividades en general } & & & 7 & 4 \\
\hline \multicolumn{2}{|l|}{ Estado de ánimo } & & & 5 & 5 \\
\hline \multicolumn{2}{|l|}{ Capacidad de caminar } & & & 7 & 4 \\
\hline \multicolumn{2}{|l|}{ Trabajo habitual } & & & 7 & 4 \\
\hline \multicolumn{2}{|l|}{ Relaciones sociales } & & & 7 & 7 \\
\hline \multicolumn{2}{|l|}{ Descanso } & & & 5 & 6 \\
\hline \multicolumn{2}{|l|}{ Ocio } & & & 7 & 6 \\
\hline \multicolumn{6}{|c|}{ Percepción de su causa } \\
\hline \multicolumn{2}{|c|}{ Enfermedad distinta a la ERCA } & & & \multicolumn{2}{|c|}{$21(23 \%)$} \\
\hline \multicolumn{2}{|l|}{ Enfermedad principal } & & & \multicolumn{2}{|c|}{$53(57 \%)$} \\
\hline \multicolumn{2}{|l|}{ Ambas } & & & \multicolumn{2}{|c|}{$19(20 \%)$} \\
\hline \multicolumn{2}{|l|}{ Situaciones } & \multicolumn{2}{|c|}{ Alivio } & Empeoramient & \\
\hline \multicolumn{2}{|l|}{ Postura } & \multicolumn{2}{|c|}{$20(22 \%)$} & \multicolumn{2}{|c|}{$5(5 \%)$} \\
\hline \multicolumn{2}{|l|}{ Calor } & \multicolumn{2}{|c|}{$11(12 \%)$} & \multicolumn{2}{|c|}{$3(3 \%)$} \\
\hline \multicolumn{2}{|l|}{ Frío } & \multicolumn{2}{|r|}{-} & \multicolumn{2}{|c|}{$9(10 \%)$} \\
\hline \multicolumn{2}{|l|}{ Reposo } & \multicolumn{2}{|c|}{$31(33 \%)$} & \multicolumn{2}{|c|}{$1(1 \%)$} \\
\hline \multicolumn{2}{|l|}{ Actividad } & \multicolumn{2}{|r|}{ - } & \multicolumn{2}{|c|}{$35(38 \%)$} \\
\hline \multicolumn{2}{|l|}{ Medicación } & $10(1$ & $1 \%)$ & & - \\
\hline Fricción/masaje & & $13(1$ & $4 \%)$ & & - \\
\hline No sabe & & & $8 \%)$ & $40(43 \%$ & \\
\hline
\end{tabular}

En la intensidad: $(0-4)=$ Leve, $(5-7)=$ Moderada, $(8-10)=$ Severa.

En la repercusión en las actividades diarias: 0=mínima repercusión, 10=máxima repercusión. 
En la Tabla 2 se representa la intensidad del dolor percibido en la última semana y la percepción que el paciente tiene, de la causa que origina el dolor.

En el momento de la entrevista (Intensidad Actual) un $38 \%$ de los pacientes no tenía dolor y un $17 \%$ una intensidad de 2. Las actividades en general, la capacidad de caminar, el trabajo habitual, las relaciones sociales y el ocio fueron las AVD en las que el dolor más repercusión tuvo.

El $69 \%$ del dolor de origen músculo-esquelético y el $73 \%$ de los calambres son los percibidos como más dolorosos (intensidad de 8 a 10).

En la Tabla 3 se recogen las estrategias de alivio del dolor, y como puede apreciarse, el reposo es la medida que mayor alivio del dolor produce; siendo el masaje la medida más eficaz en aqueIlos pacientes que presentan calambres. Llama la atención que excepto en el dolor gastro-intestinal y en las cefaleas, la medicación no es considerada la mejor medida para el dolor.

En la Tabla 4 se aprecia que un $60 \%$ de pacientes toma habitualmente mediación para el dolor, frente a un 40\% que no toma. También se describen los resultados de la opinión sobre la analgesia que tenían pautada (eficacia, efectos secundarios,...). Algunos pacientes justificaban estas repuestas al sentirse preocupados por tomar demasiada analgesia, al ser pacientes polimedicados.

Además, con este cuestionario (CBD), se encontró relación estadística entre la Intensidad Actual, el Índice de Katz ( $r=0.401, p<0.01)$, el ICC $(r=0.214, p<0.05)$, el tiempo en HD ( $r=0.237$, $p<0.05)$ y la edad $(r=0.210, p<0.05)$. También se encontró correlación entre la Intensidad Actual y la Intensidad Máxima $(r=0.288, p<0.01)$.

En cuanto al cuestionario $C D E$, en las Tablas 5 y $\mathbf{6}$ se representa la caracterización del dolor de los pacientes estudiados.

La Intensidad Total de este cuestionario, presentó una media de $8.91 \pm 2.19$. En el momento de la entrevista,
Tabla 4. Medicación pautada y opinión sobre la misma.

\begin{tabular}{|c|c|c|c|}
\hline \multicolumn{4}{|l|}{ Fármacos } \\
\hline \multicolumn{3}{|c|}{$\begin{array}{l}\text { Analgésicos no opioides } \\
\text { (paracetamol, metamizol) } \\
\text { Analgésicos opioides débiles } \\
\text { Analgésicos opioides potentes (morfina) }\end{array}$} & $\begin{array}{r}53(82 \%) \\
8(12 \%) \\
4(6 \%)\end{array}$ \\
\hline \multicolumn{4}{|l|}{ Frecuencia de la toma } \\
\hline \multicolumn{2}{|l|}{$\begin{array}{l}\text { Regularmente } \\
\text { Por necesidad }\end{array}$} & & $\begin{array}{l}30(46 \%) \\
35(54 \%)\end{array}$ \\
\hline \multicolumn{4}{|l|}{ Dosis diaria } \\
\hline \multicolumn{2}{|l|}{$\begin{array}{l}\text { No todos los días } \\
\text { 1-2 veces/día } \\
3-4 \text { veces/día } \\
5-6 \text { veces/día }\end{array}$} & & $\begin{array}{r}23(35 \%) \\
21(32 \%) \\
18(28 \%) \\
3(5 \%)\end{array}$ \\
\hline \multicolumn{4}{|l|}{ Efecto de la medicación (horas) } \\
\hline \multicolumn{3}{|l|}{$\begin{array}{l}\text { No le ayuda nada } \\
2 \text { horas } \\
3 \text { horas } \\
4 \text { horas } \\
\text { De } 5 \text { a } 12 \text { horas } \\
\text { Más de } 12 \text { horas }\end{array}$} & $\begin{array}{r}4(6 \%) \\
9(14 \%) \\
8(12 \%) \\
21(32 \%) \\
11(17 \%) \\
12(19 \%)\end{array}$ \\
\hline \multirow[t]{2}{*}{ Grado de mejoría de la medicación } & \multicolumn{2}{|c|}{ 7(Mediana) } & $4(\mathrm{RI})$ \\
\hline & Sí & No & No lo sé \\
\hline Necesita medicación más fuerte & $23(35 \%)$ & $28(43 \%)$ & $14(22 \%)$ \\
\hline Necesita más dosis & $14(21 \%)$ & $29(45 \%)$ & $22(34 \%)$ \\
\hline $\begin{array}{l}\text { Preocupado/a por tomar demasiada } \\
\text { analgesia }\end{array}$ & $23(35 \%)$ & $29(45 \%)$ & $13(20 \%)$ \\
\hline $\begin{array}{l}\text { Tiene preocupación por los efectos } \\
\text { secundarios a la medicación }\end{array}$ & $7(10 \%)$ & $58(90 \%)$ & \\
\hline Más información sobre analgesia & $33(51 \%)$ & $32(49 \%)$ & \\
\hline
\end{tabular}

la escala EVA obtuvo una mediana de 2 . El $38 \%$ de los pacientes manifestaron no tener dolor, y un $26 \%$ lo calificaron como molesto.

Cuando se analizó la Intensidad Total por centros de diálisis, los pacientes del CC presentaron mayor intensidad que los pacientes de los otros centros $(9.38 \pm 2.55$, frente a $8.69 \pm 1.65$ en el CPP y $8.44 \pm 2.08$ en la UH), 
Tabla 5. Cuestionario de Dolor Español (CDE).

\begin{tabular}{lcc} 
& Mediana & RI \\
EVA (0/10) & 2 & 5 \\
\hline Valor de Intensidad Actual (0/5) & 1 & 2 \\
\hline Intensidad Total (0/14) & 9 & 4 \\
\hline El valor de la Intensidad Total es la suma de la Intensidad Sensorial \\
(mediana de 5), la Intensidad Afectiva (mediana de 3) y la Intensidad \\
Evaluativa (valor constante=1).
\end{tabular}

Tabla 6. Frecuencias CDE.

\begin{tabular}{|c|c|c|c|}
\hline \multicolumn{2}{|l|}{ Dolor sensorial } & \multicolumn{2}{|c|}{ Dolor afectivo } \\
\hline Como pulsaciones & $15(38 \%)$ & Temible & $28(51 \%)$ \\
\hline Como una sacudida & $5(12 \%)$ & Espantoso & $14(25 \%)$ \\
\hline Como un latigazo & $20(50 \%)$ & Horrible & $13(24 \%)$ \\
\hline Frío & $23(70 \%)$ & Que marea & $6(75 \%)$ \\
\hline Caliente & $8(24 \%)$ & Sofocante & $2(25 \%)$ \\
\hline Ardiente & $2(6 \%)$ & Que atormenta & $12(38 \%)$ \\
\hline Entumecimiento & $14(16 \%)$ & Mortificante & $1(3 \%)$ \\
\hline Como un pellizco & $5(6 \%)$ & Violento & $19(59 \%)$ \\
\hline Agarrotamiento & $25(28 \%)$ & Extenuante & - \\
\hline Calambre & $20(23 \%)$ & Agotador & $20(32 \%)$ \\
\hline Espasmo & $4(4 \%)$ & Incapacitante & $43(68 \%)$ \\
\hline Retorcijón & - & Incómodo & $72(84 \%)$ \\
\hline Opresivo & $20(23 \%)$ & Que irrita & $11(13 \%)$ \\
\hline Pinchazo & $18(27 \%)$ & Que consume & $3(3 \%)$ \\
\hline Punzante & $7(10 \%)$ & Deprimente & - \\
\hline Penetrante & $16(24 \%)$ & Agobiante & $16(30 \%)$ \\
\hline Agudo & $26(39 \%)$ & Que angustia & $10(19 \%)$ \\
\hline Pesado & $17(25 \%)$ & Que obsesiona & - \\
\hline Tirante & $25(36 \%)$ & Desesperante & $27(51 \%)$ \\
\hline Como un desgarro & $4(6 \%)$ & \multicolumn{2}{|c|}{ Dolor evaluativo } \\
\hline Tenso & $23(33 \%)$ & \multirow{5}{*}{$\begin{array}{l}\text { Momentáneo } \\
\text { Intermitente } \\
\text { Creciente } \\
\text { Constante } \\
\text { Persistente }\end{array}$} & $23(25 \%$ \\
\hline $\begin{array}{l}\text { Superficial } \\
\text { Difuso }\end{array}$ & $5(6 \%)$ & & 38 (41\%) \\
\hline Que se irradia & $40(46 \%)$ & & \\
\hline Fijo & $21(24 \%)$ & & ) \\
\hline Interno & $7(8 \%)$ & & $?$ \\
\hline Profundo & $14(16 \%)$ & \multicolumn{2}{|l|}{ Dolor actual } \\
\hline Adormecido & $19(35 \%)$ & Sin dolor & $35(38 \%)$ \\
\hline Picor & $2(4 \%)$ & Leve & $20(21 \%)$ \\
\hline Hormigueo & $14(25 \%)$ & Molesto & $23(25 \%)$ \\
\hline Como agujetas & $2(3 \%)$ & Intenso & $11(12 \%)$ \\
\hline Escozor & - & Fuerte & $3(3 \%)$ \\
\hline Como una corriente & $18(33 \%)$ & Insoportable & $1(1 \%)$ \\
\hline
\end{tabular}

sin que estas diferencias fueran significativas. Una vez conocidas las frecuencias de las tres esferas: sensorial, afectiva y evaluativa; quisimos caracterizar la primera y la tercera esfera en función del tipo de dolor. Así, encontramos que:
El dolor de origen músculo-esquelético fue descrito como pulsaciones, como si la zona estuviese agarrotada, penetrante, pesado, tenso, profundo, fijo y de recurrencia constante.

El dolor relacionado con el acceso vascular principalmente fue descrito como un dolor frío, entumecido, adormecido y momentáneo.

De los tres tipos de dolor relacionados con el procedimiento, los calambres fueron los mejor caracterizados, describiéndolos como un latigazo, de carácter agudo, tirante, como una corriente, que se irradia por todo el miembro y momentáneo.

El dolor de origen isquémico fue descrito como frío, entumecido, pesado, como un pinchazo y de temporalidad intermitente.

Cuando se buscaron relaciones estadísticas entre las variables de este cuestionario (CDE), se encontró correlación entre la Intensidad Total, el Índice de Katz $(r=0.269, p<0.01)$ y el Valor de la Intensidad Actual $(r=0.318, p<0.01)$. Entre el Valor de la Intensidad Actual, el Índice de Katz $(r=0.383, p<0.01)$ y la edad $(r=0.239, p<0.05) ; y$ entre el Valor de la Intensidad Actual y la EVA $(r=0.971, p<0.01)$.

Al relacionar variables de ambos cuestionarios, se encontró correlación estadísticamente significativa entre la Intensidad Máxima ( $r=0.447 ; p<0.01$ ) y la Intensidad Actual $(r=0.362, p<0.01)$ del CBD con la Intensidad Total del $C D E$; y entre el Valor de la Intensidad Actual (CDE) y la Intensidad Actual del CBD ( $r=0.971$, $\mathrm{p}<0.01$ ).

No se encontraron más correlaciones estadísticas.

\section{Discusión}

Nuestros resultados ponen de manifiesto una prevalencia del dolor del $69 \%$ en estos pacientes, dentro del rango publicado por la mayoría de los autores (50$77 \%$ ) 17-19,22,23; aunque en algún artículo se ha publicado una prevalencia del $96 \%{ }^{24}$.

Resulta Ilamativo que la prevalencia del dolor de los pacientes en HD casi doble la del dolor crónico en la población general (11\% a 64\%) $)^{25-27}$. Parece evidente, que la mayor comorbilidad junto con la edad avanzada y el dilatado tiempo en tratamiento con HD, suponen factores claves en esta mayor prevalencia del dolor ${ }^{12}$. 
En efecto, al centrarnos exclusivamente en los pacientes que presentaban dolor, observamos que el ICC, la edad y el tiempo en HD se correlacionan significativamente con mayor intensidad del dolor (Intensidad Actual del cuestionario CBD). De igual forma, el Valor de la Intensidad Actual del cuestionario CDE se correlaciona también con dependencia y edad. Como es lógico, existe una muy buena correlación entre ambas variables. Es decir, la Intensidad Actual del cuestionario $C B D$ tiene prácticamente el mismo significado que el Valor de la Intensidad Actual del CDE, aunque se miden con escalas diferentes. EI CBD ha sido más utilizado para evaluar el dolor crónico y el CDE, tanto para evaluar dolor crónico como agudo en los pacientes en $\mathrm{HD}^{29}$.

Al comparar estos datos con los de otros autores, encontramos resultados dispares; algunos autores han encontrado relación entre el dolor y una mayor edad ${ }^{18,30}$, o mayor tiempo en HD ${ }^{18,24,31}$. En nuestros pacientes, los que presentaban dolor llevan más tiempo en diálisis que los que no presentaron, aunque sin diferencias significativas, igual que lo encontrado por otros autores ${ }^{22}$. Sin embargo, otras series publicadas no han encontrado ninguna relación entre el dolor y el tiempo en diáli$\mathrm{sis}^{20,22}$. Tampoco se encontró relación entre el dolor y ser o no diabético. Si bien en nuestra muestra, al igual que en estudios previos ${ }^{22}$, había más pacientes con diabetes entre los que tenían dolor, en comparación con los que no tenían.

Esta variabilidad de resultados puede deberse, en parte, a la conceptualización de la variable dolor, ya que en algunos estudios hacen referencia al dolor como única variable 30,32 y en otros, han analizado los resultados considerando las diferentes variables dentro de cada uno de los cuestionarios ${ }^{18-20}$, tal como hemos realizado nosotros.

Con respecto al sexo, las mujeres presentaron mayor intensidad dolorosa en comparación con los hombres, igual a lo encontrado por algunos autore ${ }^{24}$, aunque en otros estudios no han encontrado diferencias significativas entre ambos sexos ${ }^{18,31-33}$.

En lo que respecta al tipo de dolor, el de origen músculo-esquelético fue el más común entre nuestros pacientes, coincidente con lo descrito en otras series ${ }^{17,18,20,23}$. Los calambres se situaron como segunda causa de dolor, algo común en los pacientes en HD. Sin embargo, diferentes estudios no recogen la prevalencia de los calambres en sus muestras, al analizar el dolor crónico en HD. Además, los calambres se suelen presentar durante la sesión de diálisis y en menor medida en el domicilio del paciente, por lo que dependiendo del objetivo de cada estudio, en algunos trabajos no se ven reflejados $^{17,19}$. De ahí la dificultad de comparar su prevalencia e intensidad con lo publicado por otros autores ${ }^{18}$. Por su parte, la cefalea no es muy prevalente en nuestra muestra, al contrario de lo encontrado por otros autores $^{22,34,35}$. En cuanto a la localización del dolor, destaca el dolor en las extremidades, principalmente en los miembros inferiores, seguido de la espalda, similar a lo publicado en otras series ${ }^{17,18,23}$.

En cuanto a la Intensidad Máxima del CBD, del peor dolor padecido en la última semana, el $66 \%$ de los pacientes calificó este dolor como severo, similar a lo encontrado por otros autores ${ }^{20,23,31}$. Sin embargo, en el momento de la entrevista, la percepción dolorosa era baja, y el $38 \%$ de los pacientes no tenían dolor en ese momento, en la línea de lo publicado en la mayoría de los estudios ${ }^{17,18,23}$. Estos resultados ponen de manifiesto una de las dificultades que tiene caracterizar un dolor que no está en el momento presente, pero que el paciente verbaliza diferido en el tiempo. En este sentido, varios autores, para diferenciar del dolor que habitualmente presenta el paciente en HD, utilizan términos como dolor intradiálisis y dolor crónico o dolor fuera de la sesión, presentando resultados independientes para uno y otro grupo ${ }^{17,18,33}$.

A esto hay que añadir, la dificultad que tiene en ocasiones diferenciar el concepto de dolor crónico del agudo en el paciente en HD, pues el dolor agudo puede ser simplemente la exacerbación de un dolor crónico que acompaña casi siempre al paciente y que en una sesión de diálisis se manifiesta intensamente. De ahí, que nosotros hayamos querido caracterizar el dolor del paciente en HD de forma conjunta, como algo que por desgracia acompaña a estos pacientes de forma cróni$\mathrm{ca}$, y no poner tanto énfasis en el momento en el que se produce; puesto que además, en ninguno de los cuestionarios aparece una pregunta específica que plantee si el paciente en HD sufre la misma o distinta frecuencia y grado de dolor, en su casa o en la sesión de HD.

Un aspecto más relevante para nosotros era analizar la repercusión que el dolor tiene en las AVD de los pacientes. En el listado de actividades recogidas por el CBD, la mayoría se encontraban afectadas con una mediana de 7, a excepción del estado de ánimo y el descanso, que fueron las menos limitadas por el dolor. Respecto al estado de ánimo, algunos pacientes atribuyen sus cambios a problemas particulares y no al dolor. En cuanto al descanso, un número importante de pacientes 
respondió que el dolor no les perturbaba en exceso el descanso porque antes de dormir tomaban medicación al respecto. Sin embargo, los resultados encontrados en algunas series exponen justamente lo contario, siendo el estado de ánimo del paciente y el descanso los aspectos sobre los que mayor repercusión tiene el dolor ${ }^{23,33}$.

Por otro lado, el $82 \%$ de los pacientes que tomaban medicación para el dolor, solía tomar analgésicos no opioides, principalmente paracetamol. Además, como estrategia de alivio del dolor, al margen de la medicación, la mayoría eligió el reposo.

En definitiva, como principal dificultad para el análisis de los resultados, tenemos que destacar el manejo de dos cuestionarios diferentes para evaluar el dolor, lo que ha complicado la interpretación del análisis estadístico y el significado de las distintas variables en cada uno de ellos.

Como consideraciones prácticas, es importante reflexionar sobre el tipo de cuestionario genérico que se puede utilizar para el análisis del dolor en los pacientes en diálisis, puesto que no existen cuestionarios específicos para estos pacientes. En nuestra experiencia, y ante los distintos resultados ofrecidos por otros autores, y según qué cuestionario, nos atrevemos a recomendar el CBD como instrumento más adecuado para evaluar el dolor en el paciente en HD por parte de enfermería. Creemos que aporta una visión más completa de las características del dolor y de su repercusión en el paciente, para a partir de ahí poder intervenir en las diferentes estrategias de alivio o manejo del dolor, que de forma casi continua padece el paciente en HD.

A la vista de estos resultados podemos concluir que la prevalencia de dolor en la muestra estudiada es alta, y que padecer dolor del paciente en HD está relacionado con mayor edad, comorbilidad y grado de dependencia. El dolor de origen músculo-esquelético es el más frecuente y localizado principalmente en miembros inferiores.

La principal causa de dolor relacionada con el procedimiento de la HD es el calambre muscular, con una percepción dolorosa por parte del paciente muy intensa. Además, el dolor tiene una repercusión directa en la mayoría de las AVD de los pacientes, a excepción del estado de ánimo y el descanso, sobre las que influye en menor medida.

\section{Agradecimientos}

Este trabajo ha obtenido el Accésit al Premio de Investigación Fundación Renal Íñigo Álvarez de Toledo Enfermería Nefrológica 2017.

\section{El autor declara que no hay conflicto de interés.}

\section{Recibido: 15 julio 2017}

Revisado: 28 julio 2017

Modificado: 1 septiembre 2017

Aceptado: 15 septiembre 2017

\section{Bibliografía}

1. Molina Linde JM, Figueroa Rodríguez J, Uribe Rodríguez AF. El dolor y su impacto en la calidad de vida y estado anímico de pacientes hospitalizados. Univ. Psychol. 2013; 12(1): 55-62.

2. Erazo $M A$, Pérez $L$, Colmenares $C C$, Álvarez $H$, Suárez I, Mendivelso F.Prevalenciaycaracterización del dolor en pacientes hospitalizados. Rev. Soc. Esp. Dolor. 2015; 22(6): 241-8.

3. González M. Dolor crónico y psicología: actualización. Rev. med. clin. Condes. 2014; 25(4): 610-7.

4. International Association for the Study of Pain. IASP Taxonomy - IASP. 1994. [Sitio web] [Consultado 7 marzo 2017] Disponible en: https://www.iasp-pain. org/files/Content/ContentFolders/Publications2/ FreeBooks/Classification-of-Chronic-Pain.pdf.

5. González Rendón C, Moreno Monsiváis MG. Manejo del dolor crónico y limitación en las actividades de la vida diaria. Rev. Soc. Esp. Dolor. 2007; 14(6): 422-7.

6. Ministerio de sanidad, servicios sociales e igualdad. Documento Marco para la mejora del abordaje del dolor en el SNS [Sitio web] Madrid; 2014 [Consultado 26 mayo 2017]. Disponible en: http:// www.msps.es/organizacion/sns/planCalidadSNS/ pdf/excelencia/CISNS_DocumentoMarcoDolor.pdf. 
7. Langley PC, Ruiz Iban MA, Molina JT, De Andrés J, Castellón JR. The prevalence, correlates and treatment of pain in Spain. J Med Econ. 2011; 14(3): 367-80.

8. Narváez Tamayo MA, Castañeda de la Lanza C, 0 Shea Cuevas GJ, Lozano Herrera J, Castañeda Martínez C. Paciente con enfermedad renal: manejo del dolor. GAM0. 2015;14(6): 335-41.

9. Murtagh FEM, Addington-Hall J, Higginson IJ. The prevalence of symptoms in end-stage renal disease: a systematic review. Adv Chronic Kidney Dis. 2007;14(1): 82-9.

10. Koncicki HM, Unruh M, Schell J0. Pain Management in CKD: A Guide for Nephrology Providers. Am J Kidney Dis. 2017; 69(3): 45160.

11. Brkovic T, Burilovic E, Puljak L. Prevalence and severity of pain in adult end-stage renal disease patients on chronic intermittent hemodialysis: a systematic review. Patient Preference Adherence. 2016; 10: 1131-50.

12. Mansilla Francisco JJ, Díez De los Ríos Cuenca F, Cabrera Azaña S, Cortés Torres J, Macías López MJ, González Castillo JA, et al. Impact of incident comorbidity on functional loss in elderly chronic kidney disease patients undergoing hemodialysis. CANNT J. 2012; 22(1): 25-9.

13. Otero A, de Francisco A, Gayoso P et al. Prevalence of chronic renal disease in Spain: results of the EPIRCE study. Nefrología. 2010; 30(1): 78-86.

14. Sociedad Española de Nefrología. Informe de Diálisis y Trasplante [Sitio web]. Burgos: SEN; 2016. [Consultado 23 octubre 2017] Disponible en: http://www.senefro.org/contents/ webstructure/InformeREER_2016_BURGOS.pdf.

15. Phongtankuel V, Amorapanth PX, Siegler EL. Pain in the Geriatric Patient with Advanced Chronic Disease. Clin Geriatr Med. 2016; 32(4): 651-61.

16. Bailie GR, Mason NA, Bragg-Gresham JL, Gillespie BW, Young EW. Analgesic prescription patterns among hemodialysis patients in the DOPPS: potential for underprescription. Kidney International. 2004; 65(6): 2419-25.
17. Pelayo Alonso R, Martínez Álvarez $P$, Cobo Sánchez $\mathrm{JL}$, Gándara Revuelta M, Ibarguren Rodríguez E. Evaluación del dolor y adecuación de la analgesia en pacientes en tratamiento con hemodiálisis. Enferm Nefrol. 2015;18(4): 253-9.

18. Calls J, Rodríguez MA, Hernández $D$, Gutiérrez MJ, Amer FJ, Tura D, et al. Evaluación del dolor en hemodiálisis mediante diversas escalas de medición validadas. Nefrologia. 2009;29(3): 236-43.

19. Ghonemy TA, Allam HM, Elokely AM, Kadry YA, Omar HM. Chronic pain in hemodialysis patients: Role of bone mineral metabolism. Alexandria Journal of Medicine. 2016;52(4): 337-42.

20. Davison SN. Pain in hemodialysis patients: prevalence, cause, severity, and management. American Journal of Kidney Diseases. 2003;42(6): 1239-47.

21. Hsu H-J, Yen C-H, Hsu K-H, Wu I-W, Lee C-C, Hung $M-J$, et al. Factors associated with chronic musculoskeletal pain in patients with chronic kidney disease. BMC nephrology. 2014;15: 6.

22. Golan E, Haggiag I, Os P, Bernheim J. Calcium, parathyroid hormone, and vitamin $\mathrm{D}$ : major determinants of chronic pain in hemodialysis patients. Clinical journal of the American Society of Nephrology: CJASN. 2009;4(8): 1374-80.

23. Gamondi C, Galli N, Schönholzer C, Marone C, Zwahlen H, Gabutti L, et al. Frequency and severity of pain and symptom distress among patients with chronic kidney disease receiving dialysis. Swiss Med Wkly. 2013;143: w13750.

24. Özyigit S, Yıldırım Y, Karaman E. Pain in Hemodialysis Patients. Turk Neph Dial Transpl 2016; 25 (1): 88-94.

25. Goldberg DS, McGee SJ. Pain as a global public health priority. BMC public health. 2011;11: 770.

26. International association for the study of pain. How Prevalent Is Chronic Pain?. 2003. [Consultado 16 mayo 2017] Disponible en: https://www.iasp-pain. org/files/Content/ContentFolders/Publications2/ PainClinicalUpdates/Archives/PCU032_1390265045864_38.pdf.

27. Johannes CB, Le TK, Zhou $X$, Johnston JA, Dworkin $\mathrm{RH}$. The Prevalence of Chronic Pain in United States Adults: Results of an Internet-Based Survey. The Journal of Pain. 2010;11(11): 1230-9. 
28. Azevedo LF, Costa-Pereira A, Mendonça L, Dias CC, Castro-Lopes JM. Epidemiology of Chronic Pain: A Population-Based Nationwide Study on Its Prevalence, Characteristics and Associated Disability in Portugal. The Journal of Pain. 2012;13(8): 773-83.

29. Upadhyay C, Cameron K, Murphy L, Battistella $M$. Measuring pain in patients undergoing hemodialysis: a review of pain assessment tools. Clin Kidney J. 2014;7(4): 367-72.

30. El Harraqui R, Abda N, Bentata Y, Haddiya I. Evaluation and analysis of pain in chronic hemodialysis. Néphrologie \& Thérapeutique. 2014;10(7): 500-6.

31. Davison SN, Jhangri GS. The impact of chronic pain on depression, sleep, and the desire to withdraw from dialysis in hemodialysis patients. Journal of Pain and Symptom Management. 2005;30(5): 465-73.
32. Kafkia T, Vehviläinen-Julkunen $\mathrm{K}$, SapountziKrepia D. Assessment and management of pain in hemodialysis patients: A pilot study. Progress in Health Sciences. 2014;4(1): 53-60.

33. Harris TJ, Nazir R, Khetpal P, Peterson RA, Chava $P$, Patel SS, et al. Pain, sleep disturbance and survival in hemodialysis patients. Nephrol Dial Transplant 2012;27(2): 758-765.

34. Stojimirovic B, Milinkovic M, Zidverc-Trajkovic J, Trbojevic-Stankovic J, Maric I, Milic M, et al. Dialysis headache in patients undergoing peritoneal dialysis and hemodialysis. Ren Fail. 2015;37(2): 241-4.

35. Yesil S, Karslı B, Kayacan N, Süleymanlar G, Ersoy $F$. Pain evaluation in patients with chronical renal failure undergoing hemodialysis. Agri: Agri (Algoloji) Dernegi'nin Yayin Organidir = The Journal of the Turkish Society of Algology. 2015;27(4): 197-204.

Este artículo se distribuye bajo una Licencia Creative Commons Atribución-NoComercial 4.0 Internacional. https://creativecommons.org/licenses/by-nc/4.0/ 